\title{
Spatiotemporal analysis and hotspots detection of COVID-19 using geographic information system (March and April, 2020)
}

\author{
Mohsen Shariati ${ }^{1,2} \cdot$ Tahoora Mesgari $^{3} \cdot$ Mahboobeh Kasraee $^{3} \cdot$ Mahsa Jahangiri-rad $^{4,5}$
}

Received: 26 July 2020 / Accepted: 7 October 2020 / Published online: 12 October 2020

(C) Springer Nature Switzerland AG 2020

\begin{abstract}
Understanding the spatial distribution of coronavirus disease 2019 (COVID-19) cases can provide valuable information to anticipate the world outbreaks and in turn improve public health policies. In this study, the cumulative incidence rate (CIR) and cumulative mortality rate (CMR) of all countries affected by the new corona outbreak were calculated at the end of March and April, 2020. Prior to the implementation of hot spot analysis, the spatial autocorrelation results of CIR were obtained. Hot spot analysis and Anselin Local Moran's $I$ indices were then applied to accurately locate high and low-risk clusters of COVID-19 globally. San Marino and Italy revealed the highest CMR by the end of March, though Belgium took the place of Italy as of 30th April. At the end of the research period (by 30th April), the CIR showed obvious spatial clustering. Accordingly, southern, northern and western Europe were detected in the high-high clusters demonstrating an increased risk of COVID-19 in these regions and also the surrounding areas. Countries of northern Africa exhibited a clustering of hot spots, with a confidence level above 95\%, even though these areas assigned low CIR values. The hot spots accounted for nearly $70 \%$ of CIR. Furthermore, analysis of clusters and outliers demonstrated that these countries are situated in the low-high outlier pattern. Most of the surveyed countries that exhibited clustering of high values (hot spot) with a confidence level of 99\% (by 31st March) and 95\% (by 30th April) were dedicated higher CIR values. In conclusion, hot spot analysis coupled with Anselin local Moran's I provides a scrupulous and objective approach to determine the locations of statistically significant clusters of COVID-19 cases shedding light on the high-risk districts.
\end{abstract}

Keywords COVID-19 · Cumulative incidence rates (CIR) · Cumulative mortality rate (CMR) $\cdot$ Hot/cold spots · Spatial analysis

\section{Introduction}

The "novel coronavirus-infected pneumonia (NCIP)" with unknown causes was reported to the World Health Organization

Mahsa Jahangiri-rad

m.Jahangiri@iautmu.ac.ir

1 College of Engineering, Faculty of Environment, Department of Environmental Planning, Management and Education, University of Tehran, Tehran, Iran

2 Student Scientific Research Center (SSRC), Tehran University of Medical Sciences, Tehran, Iran

3 Department of Environmental Health Engineering, School of Public Health, Tehran University of Medical Sciences, Tehran, Iran

4 Department of Environmental Health Engineering, School of Health and Medical Engineering, Tehran Medical Sciences, Islamic Azad University, Tehran, Iran

5 Water Purification Research Center, Tehran Medical Sciences, Islamic Azad University, Tehran, Iran
(WHO) by Wuhan Municipal Health Commission in late December 2019 [1]. The disease is called as Corona Virus Disease 2019 (COVID-19) and its etiological agent was officially named SARS-CoV-2 (Severe Acute Respiratory Syndrome Coronavirus 2; after being provisionally called $\mathrm{HCoV}-19$ ), which was found to have $75-80 \%$ nucleotide identity to SARS-CoV [2]. Although coronaviruses have been acknowledged as minor human pathogens until the turn of the century, they were responsible for important outbreaks such as SARS-CoV and middle east respiratory syndrome coronavirus (MERS-CoV) infectious diseases in the last couple of decades [1,2]. Despite the low severity of COVID-19 as compared with SARS and MERS, SARS-CoV-2 affects a huge number of people in a short space of time due to its extremely high transmission rate $[1,2]$. The COVID-19 epidemic in mainland China has spread from Hubei province to the rest of China in around a month [1]. The outbreak was thence declared as a "public health emergency" in late Jan 2020 by WHO. Emergency of international concern and its status was thereafter upgraded from epidemic to pandemic on March 11 
[3]. Till 24th February, more than 28 countries were affected by COVID-19 and 80,000 confirmed cases announced; while 77,150 of the cases were recorded in china [2]. By the end of April, 158 countries all over the world reported 3,195,816 confirmed COVID-19 cases with 227,648 deaths. The number of patients with severe symptoms and death cases in the united states (US) were the most with 1,039,909 and 60,967, respectively $[4,5]$. Globally, the number of infected people with COVID-19 is 17 times that of the SARS-CoV outbreak in Guangdong, China in 2003 and 50 times that of the MERSCoV outbreak in 2012, which also became global public health concern. The high rate of COVID-19 mortality underlines the need for a greater understanding of the causes and spatial spread of disease which in turn could shed lights on the world outbreaks' prediction and consequently improve public health policies [2]. Heretofore, many researchers have applied spatial analysis tools to quantify the spatial patterns of phenomena. Kang et al. (2020) used spatial epidemic dynamics to investigate the outbreak of COVID-19 disease in china [6]. Martellucci et al. (2020) illustrated the changes in the spatial distribution of COVID-19 incidence in Italy using GIS-based maps [7]. Socio-economic and environmental aspects including population density, urban and rural settings, education level, lifestyle, the size of household and homeowners and climate conditions have been identified to affect the risk of catching the virus [8]. Similarly, several factors including age, urbanization, distance between cities and provinces, mean ambient temperature and level of literacy rate were spatially considered for the COVID-19 outbreak in Iran [9]. Boulos et al. (2020), worked on understanding widespread of SARS-CoV2 around the world through geographical tracking and mapping [4]. Other studies have also dealt with the spatial spread of SARS [10, 11] and MERS [12] on large samples of more than 100,000 cases. The Moran's $I$ test and Getis-Ord index are widely used to display the spatial distribution of a wide range of infectious diseases including hand, foot, and mouth and dengue disease $[13,14]$. Geographic mapping as well as geoinformatics play an important role in the study and control of epidemics [15]. This technique was also applied to the 2003 global outbreak of SARS [16]. The use of spatial analysis was firstly suggested by Dr. John Snow in 1854 to track the origin of a cholera outbreak. Since then it has become apparent that warily designed map can be very powerful decision support [15]. In the case of contagious disease epidemics, geographic mapping could assist public health decision makers, travelers and local populations at risk to perceptibly monitor trends and patterns that lies at the heart of the data and are frequently varying with time. In the study presented here, statistical and spatial analyses were applied to determine the spatial distribution and spatial clustering patterns of the COVID-19 incidence rate by the end of two time periods (March and April, 2020). This information can be beneficial for identifying the highrisk regions infected by SARS-CoV-2.

\section{Materials and methods}

\section{Data collection}

To estimate the incidence and mortality of COVID-19 for each country, the population data (world meters), estimated infectious rates and mortality rates (cases/100,000 population) of the world were obtained. Data of COVID -19 cases during two study periods, March (from 1st to $31 \mathrm{st}$ March) and April (from ${ }^{1 \text { st }}$ to 30th April), were collected from the JHU (Johns Hopkins University) [17] and world health organization [18].

The total number of recovered and deaths from COVID-19 provided by WHO [17] are shown in Table 1 which was further used for spatial analysis. Cumulative incidence rate (CIR) reveals the percentage of a population who gets sick in a specific duration of time [19]. Meanwhile, cumulative mortality rate (CMR), defines as the number of deaths (in general or caused by specific reason) in a group of people, is calculated based on that population per unit time [20]. The calculated CIR and CMR values in our study were entered into GIS software (version 10.6) to analyze the spatiotemporal distribution of officially reported COVID-19 cases [18].

\section{Spatial analysis of COVID-19}

\section{Spatial autocorrelation}

Spatial correlation and autocorrelation play a significant role in spatial modeling. As wide variety of methods for testing and measuring spatial autocorrelation have been improved, many geographers are keen on utilizing Moran's I which is one of the well-known spatial autocorrelations [21]. The global Moran's $I$ index value is within a range of -1.0 to +1.0 . This index which is a demonstrator of the spatial pattern is defined as: $(>0)$ clustered, $(=0)$ dispersed and $(<0)$ for random distribution [21]. In the present study, the spatial distribution pattern of COVID-19 incidence rate for each country was investigated by the global Moran's $I$ according to the eqs. $1-$ 3 [22]. The global Moran's $I$ index value must show the clustering distribution pattern to find high and low risk clusters for the further analysis.

Table 1 The number of cases, deaths, and recovered from COVID -19 during the study time

\begin{tabular}{llll}
\hline Dates & Cases & Deaths & Recovered \\
\hline 31st March & 743,966 & 36,258 & 154,084 \\
30th April & $3,195,816$ & 227,648 & 972,715 \\
\hline
\end{tabular}




$$
\begin{aligned}
& I=\frac{n}{S_{0}} \frac{\sum_{i=1}^{n} \sum_{i=1}^{n} W_{i, j} Z_{i} Z_{j}}{\sum_{i=1}^{n} Z_{i}^{2}} \\
& S_{0}=\sum_{i=1}^{n} \sum_{j=1}^{n} W_{i, j} \\
& Z=\frac{I-E(I)}{\sqrt{ } V(I)}
\end{aligned}
$$

where, $W_{i, j}$ represents the spatial weight between feature $i$ and $\mathrm{j}, \mathrm{n}$ is the total number of features, $Z_{i}=\left(X_{i}-\bar{X}\right)$ and $\mathrm{Z}$ statistic is the standardization of Moran's $I$.

\section{Hot spot analysis}

After obtaining the clustering pattern, the spots with the highest incidence called high-risk spots, were analyzed. The Getis-Ord Gi* statistic was accordingly determined for features by the hot spot analysis (Getis-Ord Gi*) tool in GIS software. The Getis-Ord Gi* statistic is calculated by comparing the local sum of the value for the feature in question and those of its neighbors to the sum of all feature values [23]. Zscore was obtained from $\mathrm{Gi}^{*}$ statistic for positive $Z$-scores; the larger $Z$-score reflects the more intensity of the clustering high values. For negative $Z$-scores, the smaller $Z$-score represents the more intense and the clustering of low values (i.e., a cold spot is obtained) [24].

\section{Anselin local Moran's I}

Anselin local Moran's I was used to provide information on regions with high or low risks. This index divides polygons into five categories including high-high, low-low (indicating a clustering pattern), low-high, high-low (indicating an outlier pattern), and not significant. High-high $(\mathrm{HH})$ reveals the areas where along with their surroundings have a high incidence of disease and, in turn, shows the high-risk clusters or hotspots. While, low-low (LL) spots, illustrate the areas in which the disease incidence is low and indicates the low-risk clusters of disease or cold spots [24]. In the present study, Anselin local Moran's I which provides stronger results than hot spot analysis was used to verify the examination of hot clusters or spots.

\section{Results and discussion}

\section{COVID-19 spatial distribution based on calculated CIR and CMR}

By the end of March, CIR ranged from 0 cases/100,000 population (for countries which were mostly located in central and south Africa) to 660.16 cases/100,000 population (San Marino). Ghana (451.15 cases/100,000 pop), Andorra (432.27 cases/ 100,000 pop), and Faroe Island (343.81 cases/100,000 pop) were the countries with the highest CIR records following San Marino (Table 2, Fig. 1a). A sharp increase in CIR was observed for the next month and it ranged from 0 cases $/ 100,000$ population to 4959.78 cases $/ 100,000$ population (Ghana), followed by San Marino (1659.24 cases/100,000 pop), Andorra (961.62 cases/ 100,000 pop), and Luxembourg (602.09cases/100,000 pop) by 30th April (Table 2, Fig. 1b). It should be noted that, by the end of March the countries with the missed information were Turks and Caicos Is, Reunion, Myanmar, Mayotte, Martinique, Isle of Man, Guernsey, Gibraltar, French Guiana, French Polynesia, Guadeloupe, Greenland, Faroe I., Bermuda, Aruba, and Jersey; whose information (cases and deaths) were included for the analysis conducted by the end of April.

According to Table 2 and Fig. 1c, the highest mortality rate (CMR) of COVID-19 took place in San Marino, Italy, Spain, Ghana, and Andorra. Although Italy recorded the highest
Table 2 Calculation of cumulative incidence rate (CIR) and cumulative mortality rate

\begin{tabular}{|c|c|c|c|c|c|c|c|}
\hline \multicolumn{4}{|l|}{ March } & \multicolumn{4}{|l|}{ April } \\
\hline Name & CIR & Name & CMR & Name & CIR & Name & CMR \\
\hline San Marino & 660.16 & San Marino & 70.73 & Ghana & 4959.78 & San Marino & 120.43 \\
\hline Ghana & 451.16 & Italy & 19.17 & San Marino & 1659.25 & Belgium & 64.72 \\
\hline Andorra & 432.28 & Spain & 15.70 & Andorra & 961.63 & Andorra & 54.36 \\
\hline Faroe Is. & 343.82 & Ghana & 14.84 & Luxembourg & 602.10 & Spain & 51.92 \\
\hline Luxembourg & 311.51 & Andorra & 7.77 & Iceland & 526.60 & Ghana & 47.49 \\
\hline Iceland & 298.91 & Netherlands & 5.04 & Germany & 519.87 & Italy & 45.78 \\
\hline Germany & 184.40 & France & 4.63 & Spain & 506.68 & United Kingdom & 38.54 \\
\hline Spain & 182.22 & Belgium & 4.43 & Qatar & 436.09 & France & 36.95 \\
\hline Switzerland & 178.81 & Switzerland & 3.47 & Belgium & 412.95 & Netherlands & 27.59 \\
\hline Italy & 168.27 & Luxembourg & 3.35 & Ireland & 410.16 & Sweden & 24.38 \\
\hline
\end{tabular}
(CMR) for top 10 affected countries by 31 st March and by 30th April 2020 

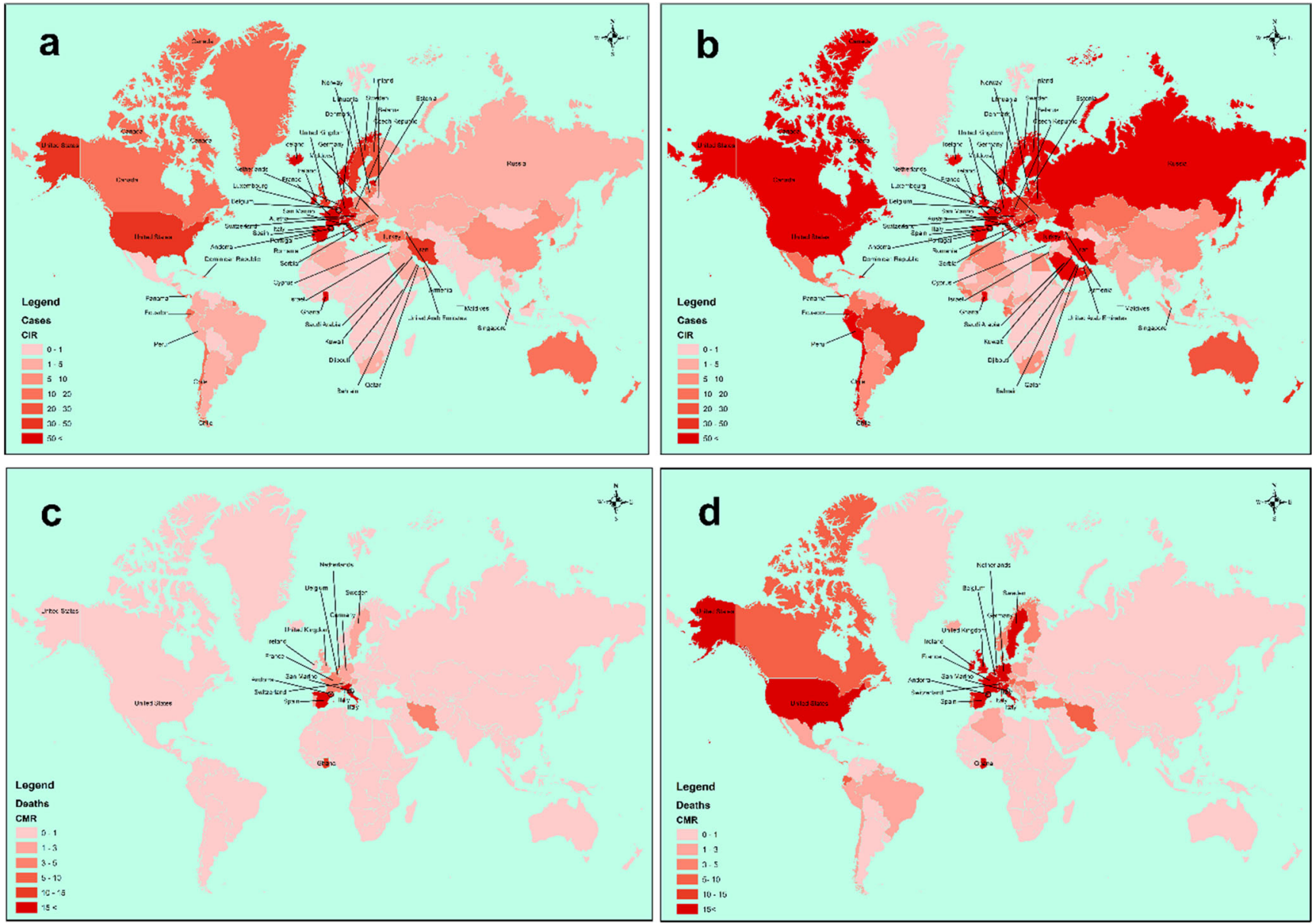

Fig. 1 Geographic distribution of CIR by the end of March (a) and April (b). Geographic distribution of CMR by the end of March (c) and April (d) for COVID -19

CMR after San Marino by 31st March, Belgium overtook the place of Italy by 30th April (Table 2, Fig. 1d).

Generally, Ghana, San Marino, Andorra, Luxembourg, Iceland, Germany, Spain, Qatar, Belgium, and Ireland recorded the highest CIR in the world. Countries with the highest CMR were San Marino, Belgium, Andorra, Spain, Ghana, Italy, United Kingdom, France, Netherland, and Sweden by the 30th April 2020, most of which are located in southern and western Europe. Among all mentioned countries, Ghana, San Marino, Andorra, Spain, and Belgium registered the highest CIR and CMR by the late of April 2020. Despite the high incidence rate of COVID-19 in Luxembourg, Iceland, Qatar, Singapore, Monaco, Liechtenstein, Bahrain, Austria, Portugal, and Austria, these countries registered low mortality rates. A possible explanation for this might be related to their demographics. Countries with younger populations are relatively healthy and contribute to the lower mortality rates. In contrast, mortality rates in most European countries and America are higher due to their elderly population [25].The capacity of each country's healthcare system to handle a rapidly spreading epidemic also accounts for these observations [26]. The global average of CIR and CMR for COVID-19 were obtained 41.12 cases $/ 100,000$ population and 3.05 deaths/100,000 population as 31st March, and 73.36 cases/ 100,000 population and 2.92 deaths $/ 100,000$ population by the end of April, respectively. The countries demonstrating the highest CIR were located from 25 degrees north latitude (Tropic of Cancer) to 65 degrees north latitude (Arctic Circle), except for Ghana. Furthermore, countries located in the African continent and the southern hemisphere had both the lowest incidence and mortality rates. Countries such as Brazil, Turkey, Russia, Iran, and China which recorded the highest cases of COVID-19 in the world, were not noticed in top 30 countries from the CIR values point of view. The higher population of mentioned communities exposed to the hazard (COVID-19) could provide an explanation for these findings which in turn decreases the CIR values.

By 31st March, 23 countries experienced the incidence rate of COVID-19 over 50 cases/100,000 population, though this number substantially increased to 56 countries by 30th April. It should also be noted that at the beginning of this study (31st March), 3 countries had recorded CMR above 15 deaths/ 100,000 population; however, 14 countries got involved by the end of April. These changes were mostly obvious for 
countries situated in southern and western Europe, as well as central and northern America, which are categorized as developed countries. According to the World Health Organization, this can be attributed to the countries' political decisionmaking [27].

\section{Evaluation of global Moran's I}

Prior to the implementation of hot spot analysis, the CIR spatial autocorrelation results were evaluated based on the feature locations and attributed values (Fig. 2). Accordingly, global Moran's $I$ statistic of spatial autocorrelation tool was exploited. The spatial autocorrelation (global Moran's I) during the study period showed that the incidence rate of COVID19 in the study area was in the clustering pattern, with the Moran's index of 0.19 and 0.02 , respectively (the respective $p$-values were less than 0.01 and less than 0.02). Based on these results the global Moran's $I$ value was close to zero (random pattern) by 30th April. The corresponding Z-score was obtained 9.50 by the end of March and there was less than $1 \%$ likelihood that this clustering pattern could be the result of random chance. However, this index decreased to 2.18 by 30th April and there was more than $\% 1$ (less than $5 \%)$ likelihood that this clustering pattern could be attributed to the random chance.

\section{Hot spot and Anselin local Moran's I analysis}

To identify COVID-19 hot-spots, the Getis-Ord Gi* statistic was applied and the results are depicted in Fig. 3(a, b). The clustering of high values (hot spots) is demonstrated in Fig. 3a with a confidence level of $99 \%$ for southern, northern and western Europe, northern Africa and a part of eastern Europe. The

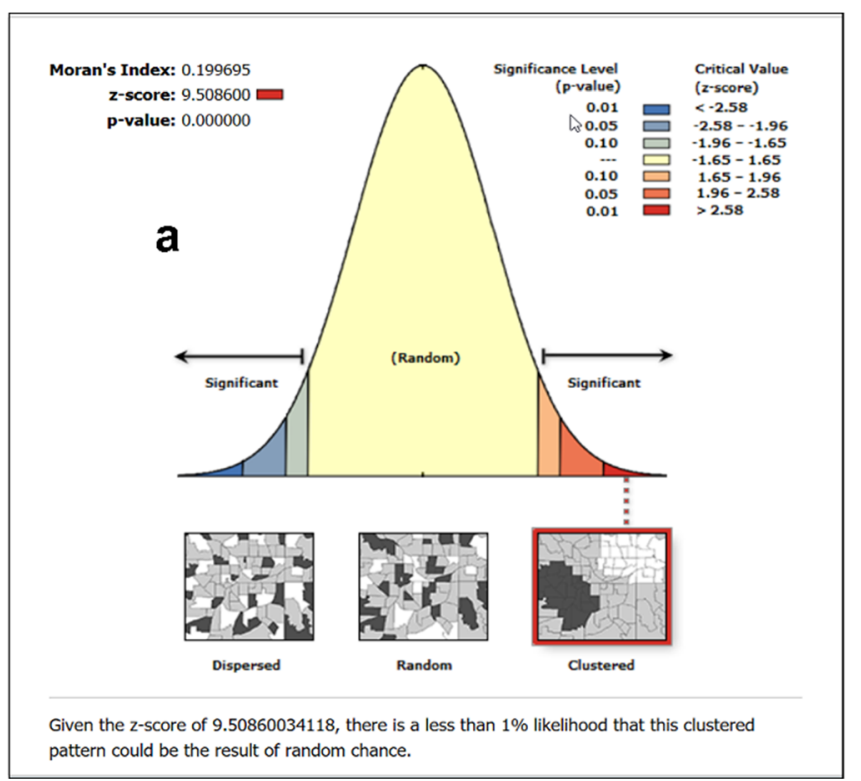

Fig. 2 Spatial autocorrelation of COVID-19 incidence rate indicator detected clustering of low values (cold spots) for eastern Africa with a confidence level of $90 \%$. Nevertheless, no clustering of the hot spot and cold spot with a confidence level of $99 \%$ was observed by the end of April (Fig. 3b). In order to determine the accuracy and significance level of the identified clusters, $p$ value and Z-score were calculated. Higher Z-scores and lower $p$ values of hot spots were identified as significantly clustered and a lower probability for being randomly selected, respectively. Consequently, cold spots with lower $Z$-scores and $p$-values were found to be clustered and less probable to be randomly selected. Likewise, a closer $Z$-score to zero shows no forms of any spatial cluster [22, 24]. Hot spot analysis (Getis-Ord GI*) is considered as a helpful tool to recognize spatial clusters of both high and low values, and has previously been applied to model several disease outbreaks $[28,29]$. Following identifying the countries in the hot spots' area, preventive measures should be taken to halt the further spreading of the virus in neighboring countries, especially those located in cold spots [30]. There is also a need to evaluate the influencing factors such as environmental, climatic, etc. components that have caused the formation of hot spot clusters in these countries [30]. Figure 4 shows the spatial distribution of the $\mathrm{Gi}^{*} Z$-scores and $p$-values, driven from the hot spot analysis. By 31st March, countries of northern Africa and a part of eastern Europe exhibited the highest value (hot spot) with a confidence level of 99\%; however, their corresponding $Z$-score values were lower than 4.8 (Fig. 4a) indicating these regions are not significantly clustered. In line with our findings, the distribution pattern of SARS-CoV epidemic in 2003 showed Guangdong province of southern China as a potential zone of disease [31]. Other countries in which chains of human-to-human transmission occurred after early importation of cases were Toronto in Canada, Hong Kong, Special Administrative Region of China, Chinese Taipei, Singapore, and Hanoi in Viet Nam [32].

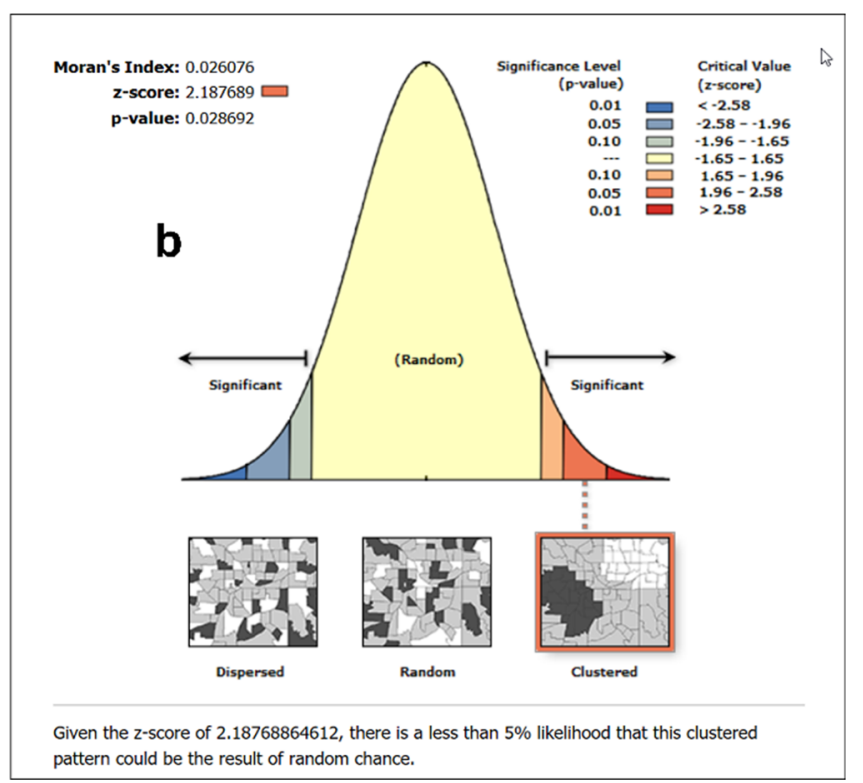




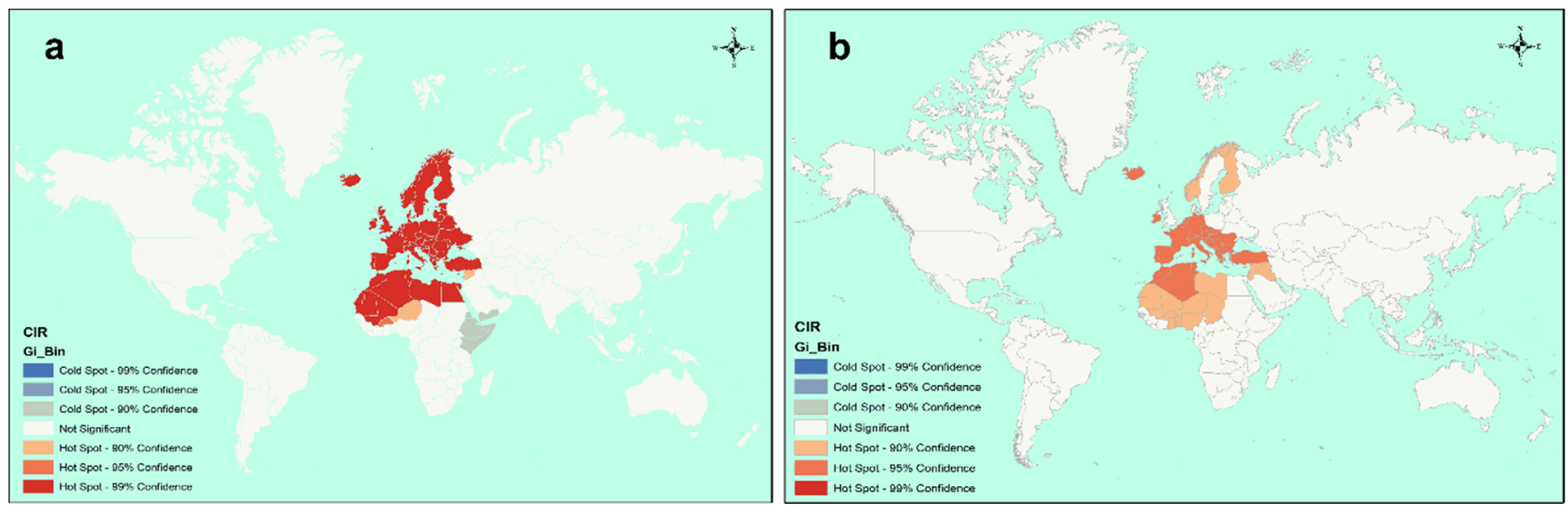

Fig. 3 Hot-spot map of COVID-19 incidence rate by (a) 31st March (b) 30th April

To determine regions with the hot and cold spots, Anselin local Moran's $I$ were used and the related maps were depicted in Fig. 5. As shown, only one high-high cluster was recognized which included southern, northern and western Europe, pointing towards an increased risk of the disease in Europe continent and the surrounding areas
(HH). It is encouraging to compare this figure with that suggested by WHO (Fig. 6) for the same period. The present findings seem to be consistent with the WHO recorded data which shows the highest degree of COVID-19 confirmed cases for Europe and the lowest observed values for Africa by 30th April 2020.
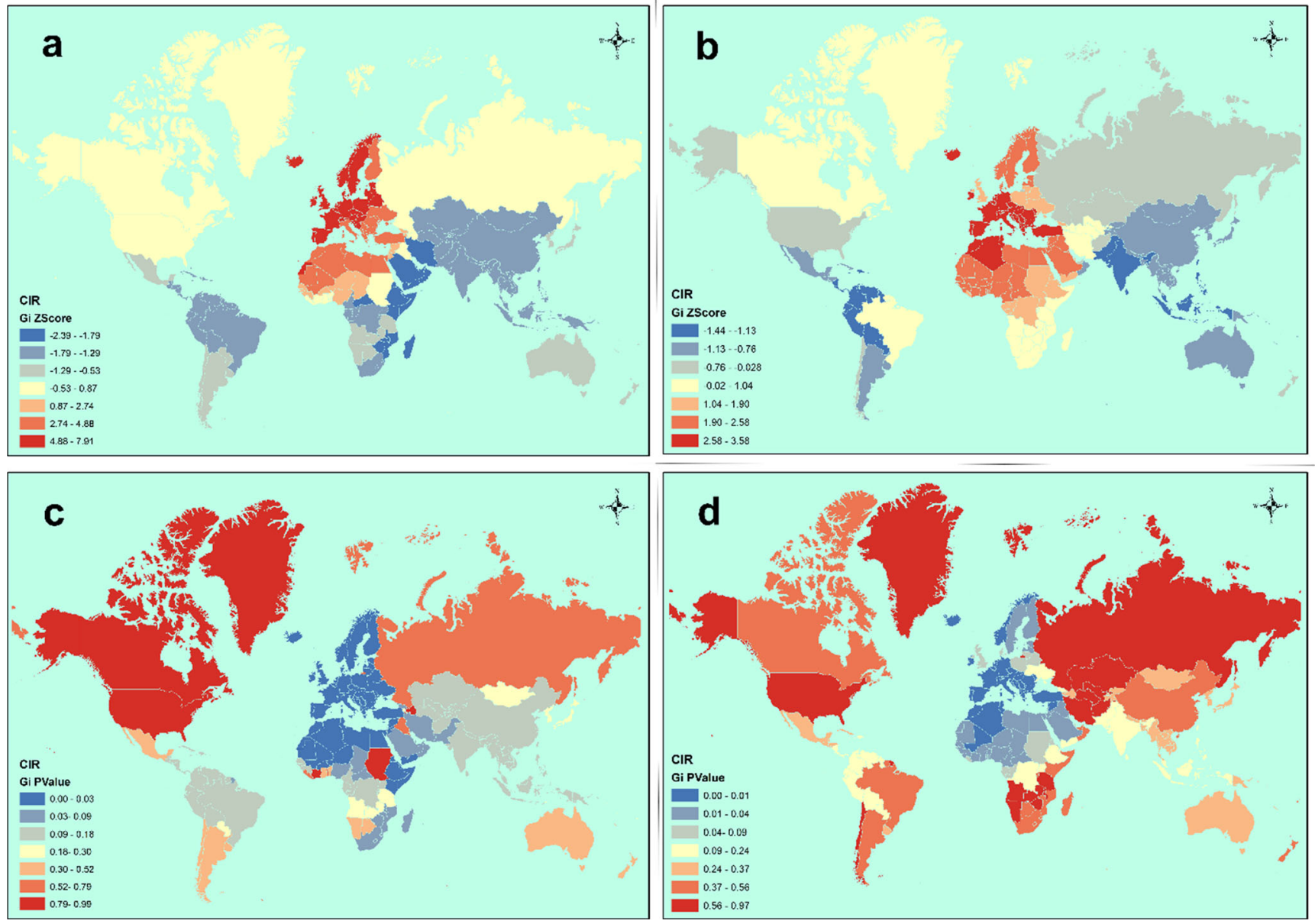

Fig. 4 Hot spot analysis of COVID-19 incidence rate. (a) $Z$-scores on 31st March (b) $Z$-scores on 30th April (c) $p$-values on 31st March and (d) $p$-values on 30th April 

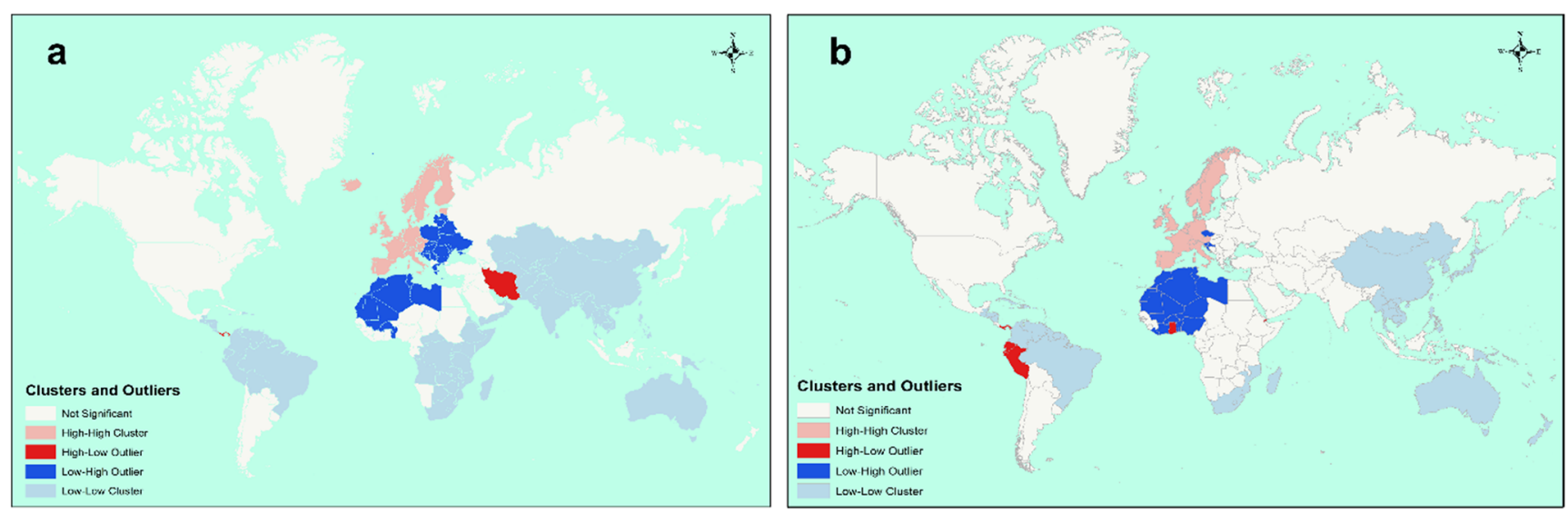

Fig. 5 Clustering analysis of COVID-19 incidence rate till (a) 31st March (b) 30th April

(a) = Americas $\equiv$ Europe $\equiv$ Sputh-East Asia $\equiv$ Eastern Mediterranean $\equiv$ Africa $\equiv$ Western Pacific 70000

60000

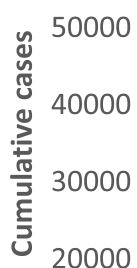

10000

0

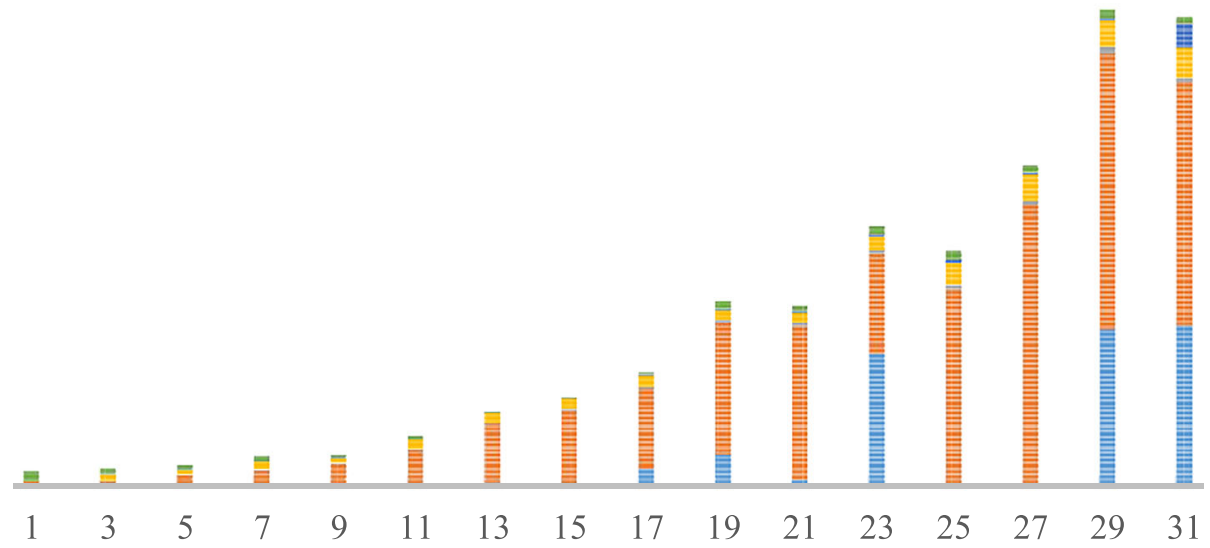

(b)

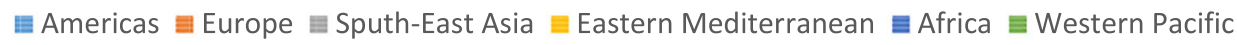
100000

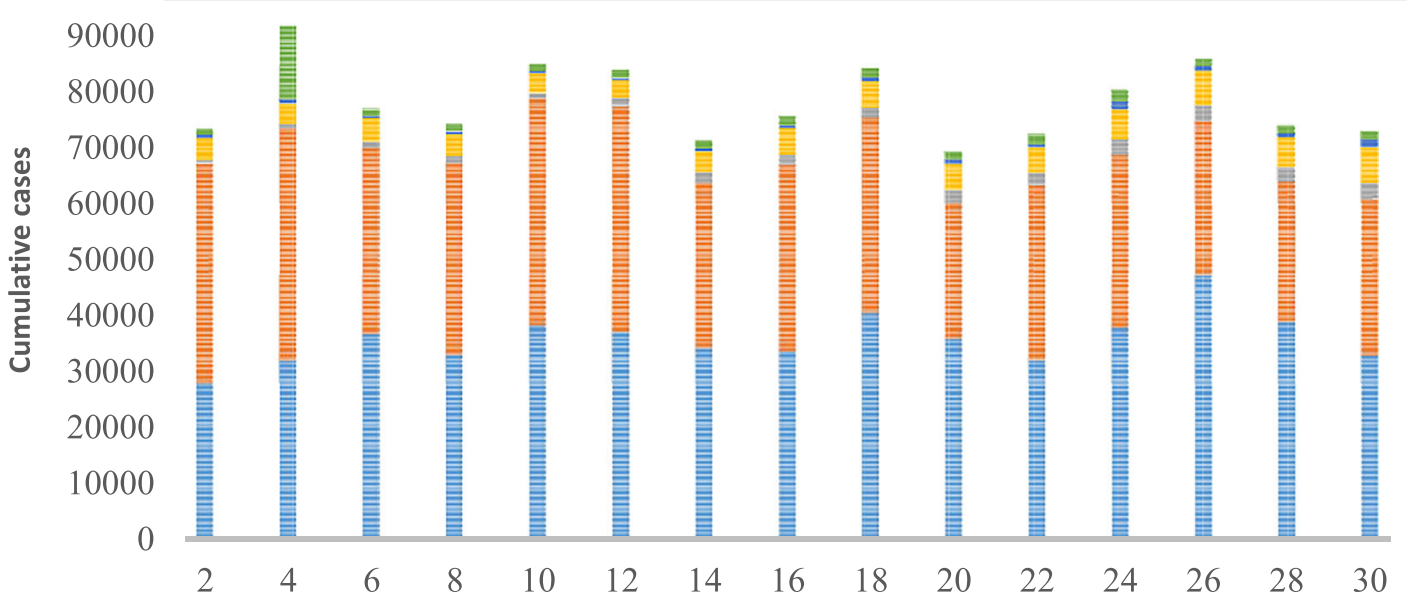

Fig. 6 Increasing trend of confirmed cases, from (a) 1st March to (b) 30th April [18] 
The chief strengths of current study is the large sample size and the analysis of geographical distribution as well as hot-spots and cold-spots of COVID- 19 epidemic. Specially, it goes beyond just clustering analysis to map the spatiotemporal variation based on CIR and CMR values by the end of March and April. The areas with hot and cold spots can help public health authorities to take preventive measures as suggested earlier [33, 34]. The output of current research and other studies which would be conducted in the future might lead to the construction of a modeling system to predict the prevalence of COVID-19 among high risk countries and to take preventive strategies as previously done regarding various infectious diseases $[35,36]$.

\section{Conclusion}

1. According to the collected data over two periods of time, the highest CIR was concentrated in southern, northern and western Europe, whereas countries located in Africa, were less affected.

2. Based on the hot spots Analysis no accurate clustered pattern was observed by 30th April (the global Moran's $I$ index value $=0.02$ )

3. According to the cluster and outlier analyses driven from Anselin Local Moran's $I$, the strong clustering of COVID19 incidence rate was detected in the southern, northern and western Europe.

4. Clusters and outliers analyses presented more accurate clustering distribution of COVID-19 in comparison with hot spot analysis, especially until 30th April.

5. Although countries in northern Africa exhibited the clustering of high values (hot spot) with a confidence level above $95 \%$, these countries ranged in low CIR. Moreover, in the analysis of clusters and outliers, this region is situated in the low-high outlier pattern surrounded by highhigh clustering pattern (southern Europe and western Europe).

Acknowledgements The authors wish to express their gratitude to Johns Hopkins University and World Health Organization whose Meta data was used in our research.

Author's contributions Conceived and designed the experiments: M. Jahangiri-rad and M. Shariati. Performed the experiments: M. Shariati. Analyzed the data: M. Shariati, M. Jahangiri-rad. Contributed reagents/ materials/analysis tools: all authors. Wrote the paper: M. Shariati, m. Jahangiri-rad.

\section{Compliance with ethical standards}

Competing interests The authors declare that they have no conflict of interests.

\section{References}

1. Surveillances $\mathrm{V}$. The epidemiological characteristics of an outbreak of 2019 novel coronavirus diseases (COVID-19) - China, 2020. China CDC Weekly. 2020;2(8):113-22.

2. Adegboye OA, Gayawan E, Hanna F. Spatial modelling of contribution of individual level risk factors for mortality from Middle East respiratory syndrome coronavirus in the Arabian Peninsula. PLoS One. 2017;12(7):e0181215.

3. Ghebreyesus TA. WHO director-General's opening remarks at the media briefing on COVID-19-11 2020. World Health Organization 2020, 11.

4. Boulos MNK, Geraghty EM. Geographical tracking and mapping of coronavirus disease COVID-19/severe acute respiratory syndrome coronavirus 2 (SARS-CoV-2) epidemic and associated events around the world: how 21 st century GIS technologies are supporting the global fight against outbreaks and epidemics. In: BioMed Central. 2020.

5. COVID C: Response Team. Geographic differences in COVID-19 cases, deaths and incidence-United States, February 12-April 7, 2020. MMWR Morb Mortal Wkly Rep. 2020;69:465-71.

6. Kang D, Choi H, Kim J-H, Choi J. Spatial epidemic dynamics of the COVID-19 outbreak in China. Int J Infect Dis. 2020;94:96-102.

7. Martellucci CA, Sah R, Rabaan AA, Dhama K, Casalone C, Arteaga-Livias $\mathrm{K}$, et al. Changes in the spatial distribution of COVID-19 incidence in Italy using GIS-based maps. Ann Clin Microbiol Antimicrob. 2020;19(1):1-4.

8. Saadat S, Rawtani D, Hussain CM. Environmental perspective of COVID-19. Sci Total Environ 2020:138870.

9. Ramírez-Aldana R, Gomez-Verjan JC, Bello-Chavolla OY. Spatial analysis of COVID-19 spread in Iran: Insights into geographical and structural transmission determinants at a province level. medRxiv 2020.

10. Lai P, Wong C, Hedley A, Lo S, Leung P, Kong J, et al. Understanding the spatial clustering of severe acute respiratory syndrome (SARS) in Hong Kong. Environ Health Perspect. 2004;112(15):1550-6.

11. Fang LQ, De Vlas SJ, Feng D, Liang S, Xu YF, Zhou JP, et al. Geographical spread of SARS in mainland China. Tropical Med Int Health. 2009;14:14-20.

12. Al-Ahmadi K, Alahmadi S, Al-Zahrani A. Spatiotemporal clustering of Middle East respiratory syndrome coronavirus (MERS-CoV) incidence in Saudi Arabia, 2012-2019. Int J Environ Res Public Health. 2019;16(14):2520.

13. Wang H, Du Z, Wang X, Liu Y, Yuan Z, Liu Y, et al. Detecting the association between meteorological factors and hand, foot, and mouth disease using spatial panel data models. Int J Infect Dis. 2015;34:66-70.

14. Tran A, Deparis X, Dussart P, Morvan J, Rabarison P, Remy F, et al. Dengue spatial and temporal patterns, French Guiana, 2001. Emerg Infect Dis. 2004;10(4):615-21.

15. Boulos MK, Roudsari AV, Carson ER. Health geomatics: an enabling suite of technologies in health and healthcare. J Biomed Inform. 2001;34(3):195-219.

16. Chan-Yeung M, Yu W. Outbreak of severe acute respiratory syndrome in Hong Kong special administrative region: case report. Bmj. 2003;326(7394):850-2.

17. (Johns Hopkins. CSSE Coronavirus COVID-19 Global Cases (dashboard). https://gisan.ddata.maps.arcgis.com/apps/ opsdashboard/index.html\#/bda7594740fd40299423467b4 8e9ecf6.

18. World Health Organization. Novel coronavirus (COVID-19) situation (public dashboard). http://healthcybermap.org/WHO COVID19/. 
19. Vandenbroucke JP, Pearce N. Incidence rates in dynamic populations. Int J Epidemiol. 2012;41(5):1472-9.

20. Porta M, editor. Mortality Rate, Morbidity rate; Death rate; Cumulative death rate; Case fatality rate. Oxford: Oxford University Press; 2014.

21. Bivand R, Anselin L, Berke O, Bernat A, Carvalho M, Chun Y, Dormann C, Dray S, Halbersma R, Lewin-Koh N: spdep: Spatial dependence: weighting schemes, statistics and models. In.: R package version 0.5-31, URL http://CRAN.R-project.org/package= spdep; 2011.

22. Martins-Melo FR, Pinheiro MCC, Ramos AN Jr, Alencar CH, de Moraes Bezerra FS, Heukelbach J. Spatiotemporal patterns of schistosomiasis-related deaths, Brazil, 2000-2011. Emerg Infect Dis. 2015;21(10): 1820-3.

23. Kim S-M, Choi Y. Assessing statistically significant heavy-metal concentrations in abandoned mine areas via hot spot analysis of portable XRF data. Int J Environ Res Public Health. 2017;14(6):654.

24. Peeters A, Zude M, Käthner J, Ünlü M, Kanber R, Hetzroni A, et al. Getis-Ord's hot-and cold-spot statistics as a basis for multivariate spatial clustering of orchard tree data. Comput Electron Agric. 2015;111:140-50.

25. Sung WYM, Robert M. Kaplan: Why Do Countries' COVID-19 Death Rates Vary So Much? 2020.

26. Iwasaki A, Grubaugh ND. Why does Japan have so few cases of COVID-19? EMBO Mol Med. 2020;12(5):e12481.

27. https://www.who.int/news-room/detail/29-06-2020-covidtimeline ToWsrtC-. Accessed 30th April.

28. Martin V, Pfeiffer DU, Zhou X, Xiao X, Prosser DJ, Guo F, et al. Spatial distribution and risk factors of highly pathogenic avian influenza (HPAI) H5N1 in China. PLoS Pathog. 2011;7(3):e1001308.
29. Firestone SM, Christley RM, Ward MP, Dhand NK. Adding the spatial dimension to the social network analysis of an epidemic: investigation of the 2007 outbreak of equine influenza in Australia. Prevent Vet Med. 2012;106(2):123-35.

30. Smith C, Skelly C, Kung N, Roberts B, Field H. Flying-fox species density-a spatial risk factor for Hendra virus infection in horses in eastern Australia. PLoS One. 2014;9(6):e99965.

31. Centers for Disease Control and Prevention (CDC): Severe acute respiratory syndrome: revised CSTE SARS Surveillance case definition. 2005.

32. health SSARSIta, https://www.who.int/ith/diseases/sars/en/.2003. Accessed 30th April.

33. Yue Y, Sun J, Liu X, Ren D, Liu Q, Xiao X, et al. Spatial analysis of dengue fever and exploration of its environmental and socioeconomic risk factors using ordinary least squares: a case study in five districts of Guangzhou City, China, 2014. Int J Infect Dis. 2018;75:39-48.

34. Azevedo L, Pereira MJ, Ribeiro MC, Soares A. Geostatistical COVID-19 infection risk maps for Portugal. Int J Health Geogr. 2020;19(1):1-8.

35. Daw MA, Shabash A, El-Bouzedi A, Dau AA. Habas M, of hepatitis LSG: Modelling the prevalence of hepatitis $C$ virus amongst blood donors in Libya: an investigation of providing a preventive strategy. World J Virol. 2016;5(1):14-22.

36. Kwong K-h, Lai P-c. Spatial components in disease modelling. In: International Conference on Computational Science and Its Applications: 2010. Springer: 389-400.

Publisher's note Springer Nature remains neutral with regard to jurisdictional claims in published maps and institutional affiliations. 